\title{
EGCG protects vascular endothelial cells from oxidative stress-induced damage by targeting the autophagy-dependent PI3K-AKT-mTOR pathway
}

\author{
Jiao Meng ${ }^{1,2 \#}$, Yuhua Chen ${ }^{1,2 \#}$, Junzhe Wang $^{2}, J_{u n l i n g}$ Qiu $^{2}$, Cuicui Chang ${ }^{2}$, Fangfang Bi ${ }^{1,2}$, Xiaopeng Wu ${ }^{2}$, \\ Wei Liu $^{2}$ \\ ${ }^{1}$ Central Laboratory of Medicine School, Xi'an Peihua University, Xi'an 710100, China; ${ }^{2}$ Department of Medical Science Research Center, Shaanxi \\ Fourth People's Hospital, Xi'an 710143, China \\ Contributions: (I) Conception and design: J Meng, Y Chen, W Liu, X Wu; (II) Administrative support: W Liu; (III) Provision of study materials \\ or patients: All authors; (IV) Collection and assembly of data: All authors; (V) Data analysis and interpretation: J Meng, Y Chen; (VI) Manuscript \\ writing: All authors; (VII) Final approval of manuscript: All authors. \\ \#These authors contributed equally to this work. \\ Correspondence to: Wei Liu, Xiaopeng Wu. Shaanxi Fourth People's Hospital, 512, East Xianning Road, Xi'an 710000, China. \\ Email: vincelau@sina.com; 149177345@qq.com.
}

Background: Autophagy plays an important role in cellular homeostasis. Epigallocatechin gallate (EGCG), a polyphenol derived from green tea, has been shown to elicit vascular protective effects. Our study aimed to investigate the protective effect of EGCG in an endothelial injury model induced by hydrogen peroxide $\left(\mathrm{H}_{2} \mathrm{O}_{2}\right)$ and reveal the possible mechanisms.

Methods: Human vascular endothelial cells (HUVECs) were pretreatment with different concentration of EGCG, then exposed to $\mathrm{H}_{2} \mathrm{O}_{2}$. Cell viability was measured with MTS assay. Apoptosis was evaluated with TUNEL staining and apoptosis-related protein was determined by western blot. Autophagy flux was assessed by transmission electron microscopy and LC3 plasmid transfection. Besides, the role mTOR in EGCGmediated antioxidant responses was validated with siRNA transfection.

Results: The results showed that pretreatment with EGCG significantly improved the survival of HUVECs from $\mathrm{H}_{2} \mathrm{O}_{2}$-induced cell death. After exposed to $\mathrm{H}_{2} \mathrm{O}_{2}$, EGCG upregulated the levels of Atg5, Atg7, LC3 II/I, and the Atg5-Atg12 complex in HUVECs, while downregulated apoptosis-related protein. Besides, EGCG inhibited the PI3K-AKT-mTOR signaling pathway. Knockdown of mTOR partially promoted EGCG-induced autophagy.

Conclusions: These results suggest that EGCG induces autophagy by targeting the mTOR pathway, indicating that EGCG has the potential to prevent and treat oxidative stress-related cardiovascular diseases.

Keywords: Epigallocatechin gallate (EGCG); autophagy; endothelial cell; mTOR; oxidative stress

Submitted Jun 22, 2019. Accepted for publication Dec 20, 2019.

doi: $10.21037 /$ atm.2020.01.92

View this article at: http://dx.doi.org/10.21037/atm.2020.01.92

\section{Introduction}

Oxidative stress plays a crucial role in the onset of cardiovascular damage and can affect a variety of related systems, leading to metabolic disorders, ischemic diseases and other diseases associated with cardiopulmonary function (1). Epigallocatechin gallate (EGCG) is a polyphenol natural product derived from green tea (Camellia sinensis). An expanding body of evidences indicate that EGCG has many biological activities, such as anti-inflammatory, antioxidant, anti-mutagenic and anti-cancer activities (2-5). Lots of work have been done to reveal the anti-cancer mechanism of EGCG. Studies have shown that EGCG 
affects multiple signaling pathways through binding to a variety of molecular targets, including transmembrane receptors, kinases and other key proteins, and ultimately leading to apoptosis, proliferation inhibition, and inhibition of invasion, angiogenesis and metastasis (6). Although it has been reported that EGCG exhibits a protective effect on the cardiovascular system (7), the protective mechanism of EGCG in oxidative stress-induced cardiovascular damage has not been fully established.

Autophagy is a defense and stress management mechanism which involves the sequestration, transport, bulk degradation and recycling of cytoplasmic components $(8,9)$. Increasing evidence indicates that autophagy is activated in oxidative stress environment (10). Recently, a lot of studies have been done on the relationship between autophagy and apoptosis, and it is demonstrated that autophagy can act as a cell survival mechanism to protect cells from apoptosis under certain conditions (2). Under oxidative stress condition, autophagy acts primarily as a pro-survival pathway to protect cardiovascular cells from oxidative damage $(7,10,11)$. Therefore, autophagy may play an important role in the healing effect of EGCG against oxidative stress.

It is well known that kinase mammalian target of rapamycin (mTOR) is a pivotal regulator of autophagy, and its activity can be enhanced by activating the PI3K/Akt signaling pathway (4). It has been verified that angiogenesis, as an important part of cardiac tissue regeneration and protection, can be regulated by mTOR (12). Besides, induction of autophagy through targeting mTOR pathway alleviates vascular smooth muscle cell senescence induced by Adriamycin (8). Further, inhibition of mTOR for prolonged treatment has been proved to be beneficial in reducing vasculopathy (13). In addition, through repression of the $\mathrm{PI} 3 \mathrm{~K} / \mathrm{AKT} / \mathrm{mTOR}$ pathway, restoring autophagy flux alleviates ox-LDL-induced human vascular endothelial cells (HUVECs) injury (14). All these evidences indicate that it is necessary to pay attention to mTOR when autophagy is investigated in cardiovascular disease.

We hypothesized that induction of autophagy may be a potential mechanism by which EGCG protects cardiovascular cells from oxidative stress damage. Herein, by examining how EGCG affects autophagy flux in endothelial cells in a dose-dependent manner, and by determining the role of mTOR in EGCG-mediated antioxidant responses, our study indicates that pretreatment with EGCG triggers autophagic survival response of endothelial cells in oxidative stress environment.

\section{Methods}

\section{Cell culture and treatment}

HUVECs were bought from ScienCell Research laboratories (USA). Cells were cultured in M199 containing 20\% fetal bovine serum (FBS) (Gibco, Carlsbad, CA, USA), $200 \mu \mathrm{g} / \mathrm{mL}$ penicillin (Gibco), $200 \mu \mathrm{g} / \mathrm{mL}$ streptomycin (Gibco) and $2 \mathrm{mM}$ L-glutamine (Thermo Scientific, Carlsbad, CA, USA) at $37{ }^{\circ} \mathrm{C}$ with $5 \% \mathrm{CO}_{2}$ (15). EGCG powder (purity $98 \%$ ) was dissolved in distilled $\mathrm{H}_{2} \mathrm{O}$ in the concentration of $10 \mathrm{mM}$, and diluted to the desired concentration. The cells were pretreated with $\operatorname{EGCG}(1,5,10 \mu \mathrm{mol} / \mathrm{L})$ for $24 \mathrm{~h}$, after which the original medium was removed and the fresh medium containing $200 \mu \mathrm{mol} / \mathrm{L} \mathrm{H}_{2} \mathrm{O}_{2}$ was added. After incubated for another $4 \mathrm{~h}$, the following tests were performed.

\section{Cell viability analysis}

Cell viability was measured with MTS assay. Briefly, HUVECs were seeded in 96-well tissue culture plates and pretreated with EGCG at various concentrations (1 to $10 \mu \mathrm{mol} / \mathrm{L}$ ) or vehicle for $24 \mathrm{~h}$, then treated with $200 \mu \mathrm{mol} / \mathrm{L}$ $\mathrm{H}_{2} \mathrm{O}_{2}$. After treatment, $10 \mu \mathrm{L}$ MTS assay solution was added to each well and incubated at $37^{\circ} \mathrm{C}$ for $3 \mathrm{~h}$. Since MTS is light-sensitive, the procedure was performed in dark environment. Then, the optical density of each well was measured at $570 \mathrm{~nm}$ with a microplate reader (Molecular Device, Sunnyvale, CA, USA). The test was performed in five wells and repeated three times.

\section{TUNEL assay}

Terminal deoxynucleotidyl transferase-mediated dUTP-biotin nick end labeling (TUNEL) staining was performed using a TUNEL Apoptosis Assay Kit (T2190, Solarbio, Beijing, China) and according to the manufacturer's instructions. The drug-treated cells were fixed with paraformaldehyde (PFA) for 15 min, gently rinsed with PBS and mixed with Triton-X-100 $(0.2 \%)$ (T8200, Solarbio) for 10 mins at room temperature. Add the newly prepared TUNEL assay solution and incubate at $37^{\circ} \mathrm{C}$ in the dark for $1 \mathrm{~h}$. Slides were rinsed with phosphatebuffer saline (PBS) three times and counterstained with $10 \mu \mathrm{g} / \mathrm{mL}$ PI (P4170, Sigma, MO, USA) at room temperature in the dark for $20 \mathrm{~min}$. The percentage of TUNEL-positive cells in 10 randomly chosen fields (85-105 cells/field) was calculated as TUNEL positive nuclei/PI staining cells, with a fluorescence microscope (Olympus BX-51). The test was performed independently at least twice. 


\section{Transmission electron microscopy (TEM)}

Cells were fixed with $2.5 \%$ glutaraldehyde overnight at $4{ }^{\circ} \mathrm{C}$, then fixed with $1 \%(\mathrm{w} / \mathrm{v})$ osmium tetroxidefor $1 \mathrm{~h}, 1 \%(\mathrm{w} / \mathrm{v})$ aqueous uranyl acetate overnight, and dehydrated in $50 \%$, $70 \%$, and $95 \%$ pure alcohol (v/v). Samples were then infiltrated and embedded using Epon resin, resin blocks were then polymerized at $70{ }^{\circ} \mathrm{C}$ for $48 \mathrm{~h} .0 .1 \mu \mathrm{m}$ ultrathin sections were stained with $5 \%$ lead citrate $[(\mathrm{w} / \mathrm{v})$, Sigma, MO, USA] and then observed using a Zeiss transmission electron microscope (Zeiss Inc., Thornwood, NY, USA) at an accelerating voltage of $80 \mathrm{kV}$.

\section{Immunoblot analysis assay}

Antibodies against AKT (4691S), p-AKT (4060S), mTOR (2983T), p-mTOR (5536T), LC3 (12741S), Atg5 (12994S), Atg7 (8558S), Atg12 (2011S), caspase 3 (9665S), cleavedcaspase $3(9664 \mathrm{~S})$, caspase 9 (9508S) and cleaved-caspase 9 (7237S) were bought from Cell Signaling Technology (CST, Danvers, MA, USA). Antibodies against 3-methyladenine (3-MA) (M9281) and $\beta$-actin (A8481) were from Sigma. Briefly, HUVECs were lysed, then quantified proteins using the BCA assay (23225, Thermo Scientific). An equal amount of protein samples were subjected to sodium dodecyl sulfate-polyacrylamide gel electrophoresis (SDSPAGE) and electro-transferred to a $0.45 \mu \mathrm{m}$ polyvinylidene difluoride membranes (Millipore, HATF09025). The membranes were blocked with $5 \%$ BSA and incubated with primary antibodies at $4{ }^{\circ} \mathrm{C}$ overnight. The membranes were then incubated with secondary antibody (CST, 1:20,000) for $1 \mathrm{~h}$ at room temperature followed with three 10-min washes in TBS-T. bands were visualized using an enhanced chemiluminescence (ECL) system. Data within a linear range were quantified using Quantity One software (BIORAD, Hercules, CA, USA).

\section{Plasmid electroporation and confocal microscopy}

Briefly, resuspend the cells in serum and antibiotic-free M199 medium at a density of $1 \times 10^{6}$ cells/mL. $200 \mu \mathrm{L}$ of cell suspension were mixed with $5 \mu \mathrm{g}$ mCherry-EGFP-LC3 plasmid, which was a gift from Prof. Xuejun Li from Peking University Health Science Center (Addgene plasmid, 22418), then transferred to a $0.4 \mathrm{~cm}$ electroporation cuvette (BioRad, Hercules, USA), and electroporated using Gene Pulser Xcell ${ }^{\mathrm{TM}}$ Electroporation System (BioRad) (16). The pulse amplitude of electroporation varied between 80 and
$120 \mathrm{~V}$ with a pulse duration of $20 \mathrm{~ms} /$ transfection. When the electroporation was over, the cells were incubated at room temperature for $5 \mathrm{~min}$ and transferred to an 8-well LAB-Tek confocal CHAMBERCVG (LAB-Tek, 155411, Hatfield, PA, USA)

After transfection, HUVECs were treated with different drugs as previously described. Immunofluorescence staining images of HUVECs transfected with mCherry-EGFPLC3 plasmid were obtained at 40× magnification using a Leica TCS SP5 laser scanning confocal microscope. The fluorescence of EGFP (green) and mCherry (red) were excited with laser light of $488 \mathrm{~nm}$ and $587 \mathrm{~nm}$, respectively.

\section{Small interfering RNA (siRNA) transfection}

The siRNAs targeting mTOR was obtained from Santa Cruz and was a pool of three target-specific 19-25 nt siRNAs. The siRNAs were transfected into HUVECs using Lipofectamine 3000 (Invitrogen) according to the manufacturer's instructions.

\section{Data analysis and statistics}

All data were presented as the means \pm SEM. Data were analyzed with GraphPad Prism 6.0 and SPSS 18.0. Statistical analysis was performed by using one-way ANOVA followed by Bonferroni correction. A value of $\mathrm{P}<0.05$ was considered statistically significant.

\section{Results}

\section{EGCG protected HUVECs from $\mathrm{H}_{2} \mathrm{O}_{2}$-induced apoptosis}

To determine the most appropriate oxidative stress conditions, HUVECs were treated with different concentrations of $\mathrm{H}_{2} \mathrm{O}_{2}$ (from $100 \mu \mathrm{mol} / \mathrm{L}$ to $1 \mathrm{mmol} / \mathrm{L}$ ). It was found that $\mathrm{H}_{2} \mathrm{O}_{2}$ obviously reduces cell viability of HUVECs in a dose-dependent manner (Figure 1A). Based on these results, treatment with $200 \mu \mathrm{mol} / \mathrm{L} \mathrm{H}_{2} \mathrm{O}_{2}$ for $4 \mathrm{~h}$ was selected to provide a maximum dynamic range for quantifying protective and deleterious responses. Besides, we demonstrated that treatment with EGCG $(0-40 \mu \mathrm{mol} / \mathrm{L})$ alone did not significantly influence cell survival, suggesting that EGCG had no toxic effect on HUVECS (Figure 1B). To evaluate if EGCG pretreatment could protect HUVECs from oxidative stress injury, an MTS assay was carried out to assess cell viability. As illustrated, cells treated with $200 \mu \mathrm{mol} / \mathrm{L} \mathrm{H}_{2} \mathrm{O}_{2}$ for $4 \mathrm{~h}$ showed typical characteristics of 
A

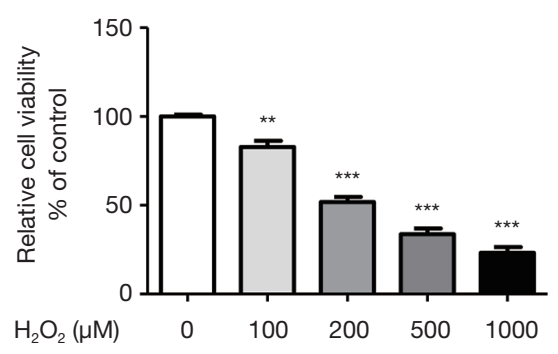

B

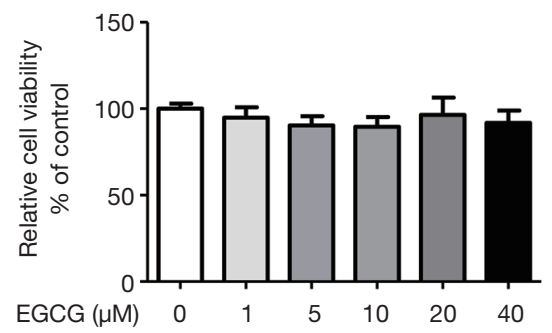

C

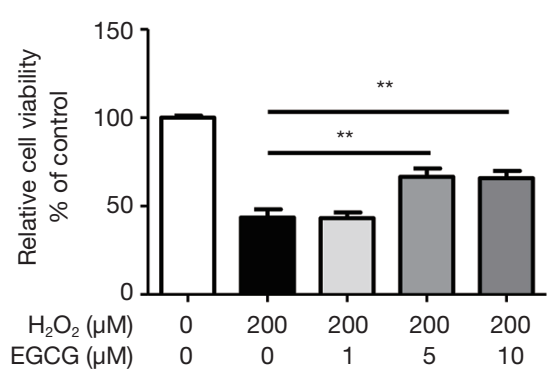

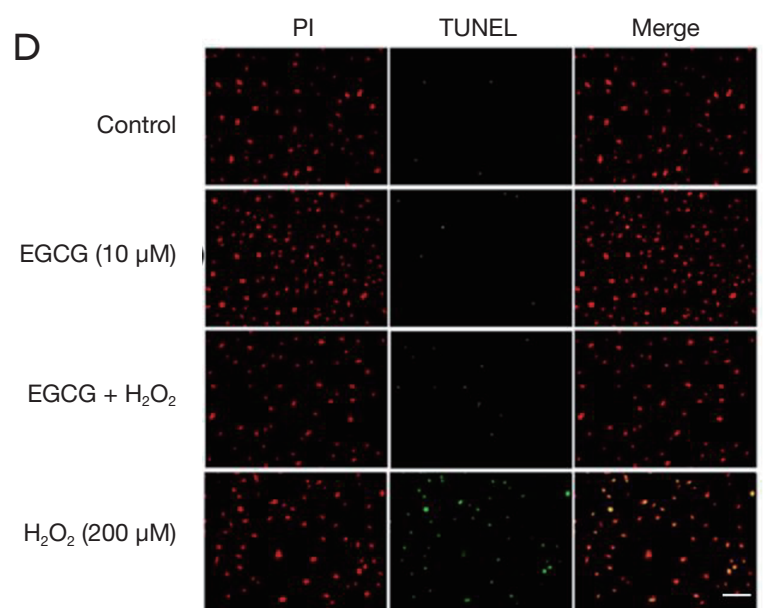

$\mathrm{E}$

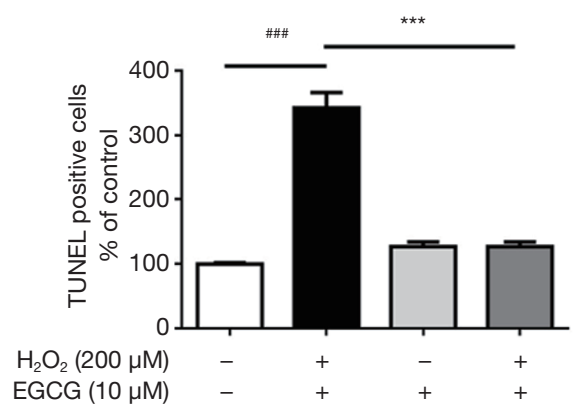

Figure 1 The protective effect of EGCG on $\mathrm{H}_{2} \mathrm{O}_{2}$-induced decreased cell viability in HUVECs. (A) HUVECs were incubated with increasing concentrations of $\mathrm{H}_{2} \mathrm{O}_{2}(100-1,000 \mu \mathrm{mol} / \mathrm{L}) . \mathrm{n}=3$. ${ }^{* *}, \mathrm{P}<0.01$, ${ }^{* * *}, \mathrm{P}<0.001 v s$. control group. (B) Effect of different concentration of EGCG on the viability of HUVECs. (C) Effect of EGCG pretreatment on the viability of HUVECs treated with $\mathrm{H}_{2} \mathrm{O}_{2}$. HUVECs were exposed to various concentrations of EGCG $(1,5,10 \mu \mathrm{mol} / \mathrm{L})$ for $24 \mathrm{~h}$. Then, cells were treated with $\mathrm{H}_{2} \mathrm{O}_{2}(200 \mu \mathrm{mol} / \mathrm{L})$ for $4 \mathrm{~h}$ and cell viability was tested by MTS assay. $\mathrm{n}=3 .{ }^{* *}, \mathrm{P}<0.01$ vs. $\mathrm{H}_{2} \mathrm{O}_{2}$ alone treatment group. (D) Representative staining with TUNEL and PI. (E) The number of TUNEL-positive nuclei was expressed as a percentage of the total nuclei detected. $n=5 .{ }^{\text {.\#\#\# }}, \mathrm{P}<0.001$ vs. control group, ${ }^{* * *}$, $\mathrm{P}<0.001 v s . \mathrm{H}_{2} \mathrm{O}_{2}$ alone group. Data are shown as the mean $\pm \mathrm{SEM}$.

apoptosis, whereas EGCG pretreatment $(5,10 \mu \mathrm{mol} / \mathrm{L})$ alleviated $\mathrm{H}_{2} \mathrm{O}_{2}$-induced damage and restored cell survival following the oxidative stress (Figure 1C). Further, results of TUNEL staining showed that the number of TUNELpositive cells in the EGCG pretreatment group was significantly lower than that of the $\mathrm{H}_{2} \mathrm{O}_{2}$ alone treatment group (Figure 1D,E).

To further determine whether the protective effect of EGCG on HUVECs was related to apoptosis in vitro, we evaluated the expression of cleaved-caspase 3 and cleavedcaspase 9 by western blot analysis. The results showed that the expression levels of cleaved-caspase 3 and cleaved- caspase 9 were significantly increased after 4 h $\mathrm{H}_{2} \mathrm{O}_{2}$ treatment compared with the control group (Figure 2A). However, EGCG pretreatment significantly reversed this upward trend. $10 \mu \mathrm{mol} / \mathrm{L}$ EGCG had a significant effect on reducing cleaved-caspase 3 and cleaved-caspase 9 in HUVECs (Figure 2B,C). These results confirm that EGCG protects HUVECs from oxidative stress-induced apoptosis.

\section{EGCG promoted autophagy in $\mathrm{H}_{2} \mathrm{O}_{2}$-treated HUVECs}

To investigate if EGCG pretreatment affected the level of autophagy, we observed HUVECs with TEM, which is the 


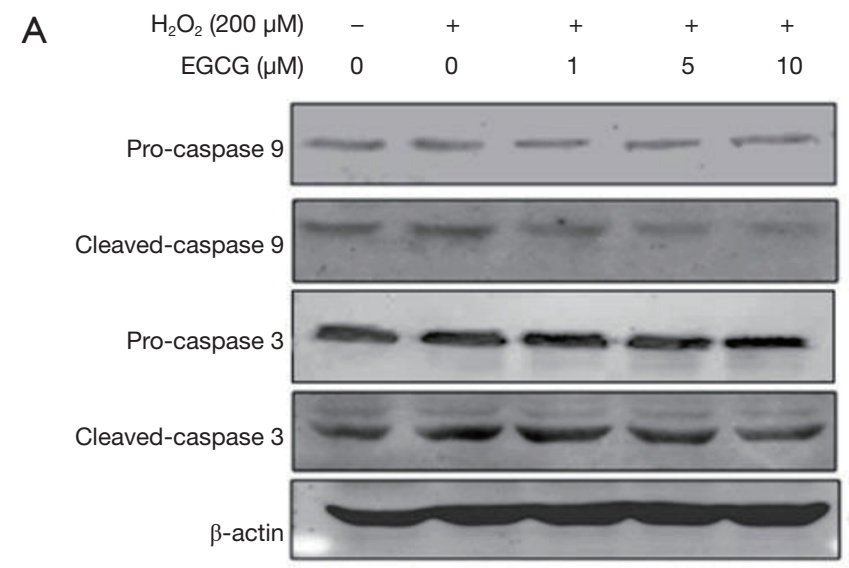

B

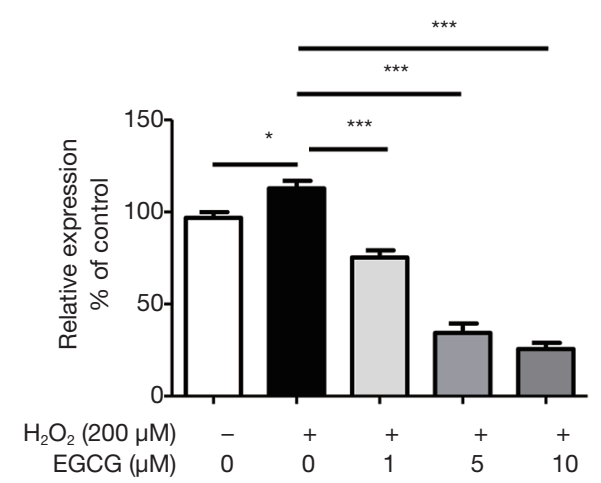

C

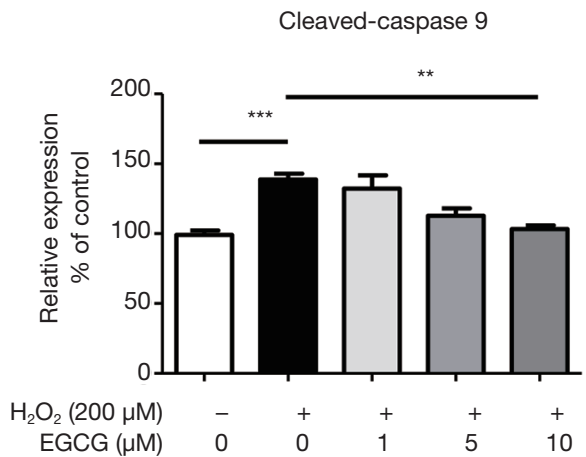

Figure 2 EGCG reversed $\mathrm{H}_{2} \mathrm{O}_{2}$-induced up-regulation of apoptosis marker proteins. (A) Representative western blot analysis of cleavedcaspase 3, cleaved-caspase 9 in EGCG (1, 5, $10 \mu \mathrm{mol} / \mathrm{L})$ pretreated HUVECs. Densitometric analysis was used to quantify the level of (B) cleaved-caspase 9 and (C) cleaved-caspase 3 activation. Values were normalized against $\beta$-actin and presented as fold increases compared with those at the basal level (control). Data are shown as the mean $\pm \mathrm{SEM}, \mathrm{n}=3 .{ }^{*} \mathrm{P}<0.05$ vs. control group, ${ }^{* *} \mathrm{P}<0.01,{ }^{* * *} \mathrm{P}<0.001$ vs. $\mathrm{H}_{2} \mathrm{O}_{2}$ alone group.

classic method for detecting autophagy in most organisms. In the control group, the HUVECs were normal. Obvious membranolysis (white arrowhead), vacuoles, apoptosis bodies (yellow arrowhead) due to oxidative stress could be seen in $\mathrm{H}_{2} \mathrm{O}_{2}$ alone treatment group. While in EGCG pretreatment group, the HUVECs were protected from $\mathrm{H}_{2} \mathrm{O}_{2}$-induced oxidative stress. HUVECs showed fewer features of apoptosis and more autophagosomes (red arrowhead indicating bilayer membrane structure) (Figure $3 A$ ). Besides, EGCG did not affect the morphology and ultrastructure of normal HUVECs cells. The TEM analysis is consistent with the TUNEL assay and Hoechst staining results.

To assess the role of EGCG in autophagy in HUVECs, mCherry-EGFP-LC3 tandem tagged fluorescent protein
(tfLC3) was transfected into HUVECs and then observed with a confocal microscopy. As previously described, the green LC3 puncta indicated autophagosomes, while the red LC3 puncta represented both autophagosomes and autolysosomes, the yellow spots, formed by the superposition of green and red, represented autophagosomes $(9,17)$. In our study, the number of green, red, and yellow puncta in EGCG $(10 \mu \mathrm{mol} / \mathrm{L})$ pretreatment group were significantly higher (Figure $3 B$ ), which suggested that EGCG enhanced autophagy flux in HUVECs.

Next, the expression levels of microtubule associated protein 1 light chain 3-II (LC3-II), which is an LC3-conjugate and an autophagosomal marker, was assessed (17). Western blot analysis indicated that the ratio of LC3-II/I significantly elevated in a dose-dependent 

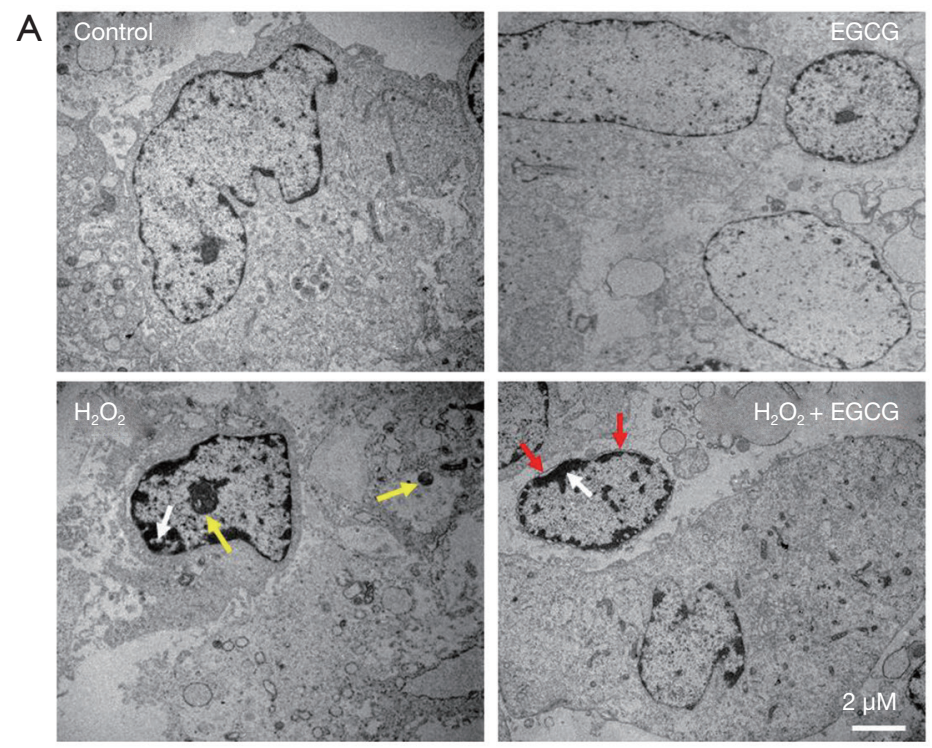

B
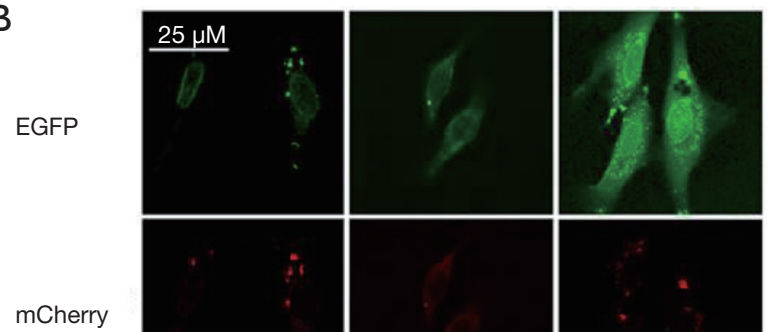

mCherry
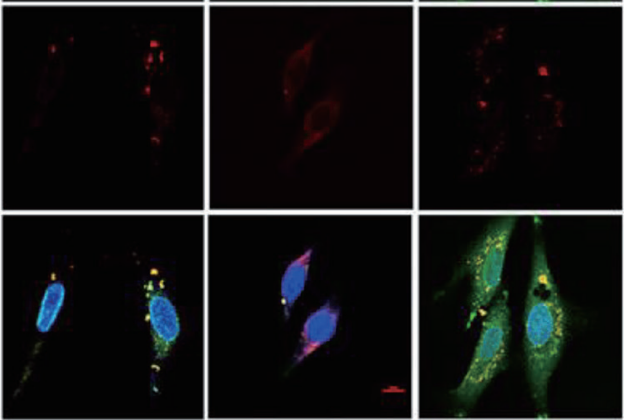

$\mathrm{H}_{2} \mathrm{O}_{2}(200 \mu \mathrm{M})$

EGCG $(10 \mu \mathrm{M})$
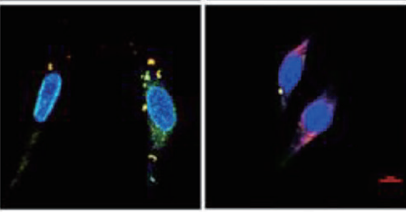

$+$

Figure 3 EGCG promoted autophagy in HUVECs. (A) TEM was used to detect cell autophagy of HUVECs pretreatment with or without EGCG and following treatment with $\mathrm{H}_{2} \mathrm{O}_{2}$. White arrowhead: membranolysis; yellow arrowhead: vacuoles and apoptosis bodies; red arrowhead: autophagosomes. The changes of autophagy activity were identified by imaging (scale bars, $2 \mu \mathrm{m}$ ). (B) Confocal images of representative images of GFP and mCherry fluorescent puncta in HUVECs transfected with mCherry-EGFP-LC3 for $24 \mathrm{~h}$ and treated with or without EGCG $(10 \mu \mathrm{mol} / \mathrm{L})$ for $24 \mathrm{~h}$, following $\mathrm{H}_{2} \mathrm{O}_{2}(200 \mu \mathrm{mol} / \mathrm{L})$ treatment for $4 \mathrm{~h}$.

manner in EGCG $(1,5,10 \mu \mathrm{mol} / \mathrm{L})$ pretreatment cells compared with $\mathrm{H}_{2} \mathrm{O}_{2}$ alone treated cells. EGCG also increased the expression of Atg5 and Atg7, which have been believed to be molecules essential for autophagy (Figure 4A,B). Consistently, when pretreated with EGCG before $\mathrm{H}_{2} \mathrm{O}_{2}$ stimulation in HUVECs, the expression of Atg5-Atg12 complex increased, and the level of P62/ SQSTM1 significantly decreased. Whereas, when given 10 $\mathrm{mM} 3-\mathrm{MA}$, an autophagy inhibitor, for $1 \mathrm{~h}$ before exposed to $\mathrm{H}_{2} \mathrm{O}_{2}$, could weaken the effect of EGCG (Figure 4C,D).

\section{EGCG downregulated the PI3K-AKT-mTOR signaling pathway in HUVECs exposed to oxidative stress}

To further determine the mechanism of EGCG-induced autophagy, we examined Ser473 phosphorylation of AKT, which measured both AKT-mTORC1 and mTORC2 activity (18). As illustrated, the levels of p-AKT, p-PI3K and p-mTOR were markedly decreased in a dose-dependent manner due to EGCG pretreatment compared with these in $\mathrm{H}_{2} \mathrm{O}_{2}$ alone treatment group (Figure $5 A, B$ ). Moreover, within $4 \mathrm{~h}$, by observing $200 \mu \mathrm{mol} / \mathrm{L} \mathrm{H}_{2} \mathrm{O}_{2}$ combined $10 \mu \mathrm{mol} / \mathrm{L}$ EGCG treatment group in terms of time, we found that p-Akt and p-PI3K were markedly reduced after
$60 \mathrm{~min}$ in HUVECs (Figure 5C,D).

\section{Knockdown of mTOR partially promoted EGCG-induced autophagy}

We used a siRNA interference vector to knock down human mTOR gene expression in HUVECs. Western blot analysis showed that the amount of mTOR protein expression was reduced by $60-70 \%$ after $48 \mathrm{~h}$ transfection; as expected, the levels of Atg5, Atg12, and Atg5-Atg12 complex were increased compared with control group transfected with nontargeting scramble siRNA transiently (Figure 6A). We detected the subcellular localization of GFP-LC3, a green fluorescent autophagy reporter protein, and the results showed that mTOR-siRNA promoted GFP-LC3 puncta formation (Figure 6B). Besides, we found that knockdown of mTOR could promoted the protective effect of EGCG (Figure 6C). Next, we evaluated if inhibition of autophagy by 3-MA affected the EGCG-induced protection in HUVECs. We pretreated the cells with EGCG for $24 \mathrm{~h}$, one sample of which was given $10 \mathrm{mmol} / \mathrm{L}$ of 3-MA, and finally injured the cells by exposed to $200 \mu \mathrm{mol} / \mathrm{L} \mathrm{H}_{2} \mathrm{O}_{2}$ for $4 \mathrm{~h}$. Compared with the EGCG $+\mathrm{H}_{2} \mathrm{O}_{2}$ groups, cell viability in EGCG + $3-\mathrm{MA}+\mathrm{H}_{2} \mathrm{O}_{2}$ treatment groups were reduced (Figure 6D). 


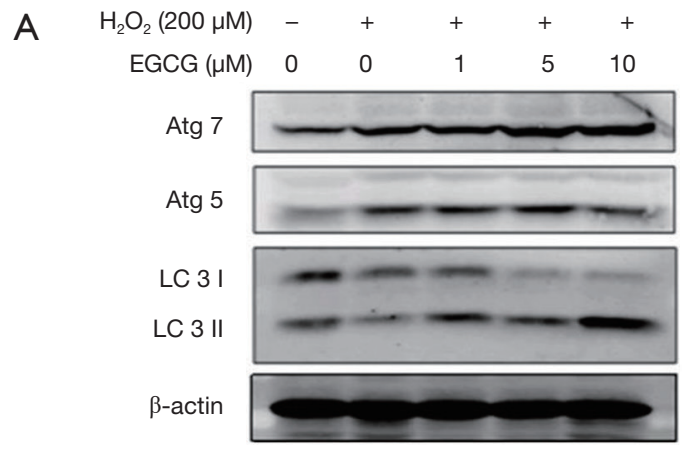

B

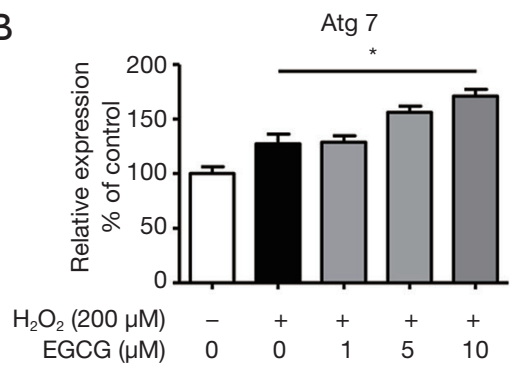

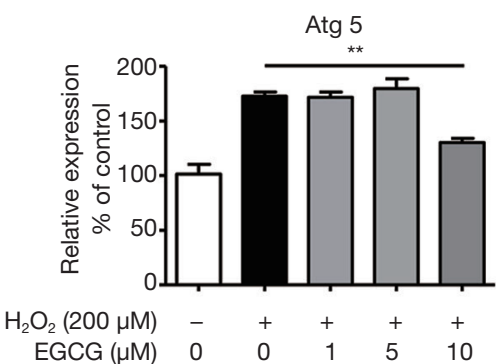

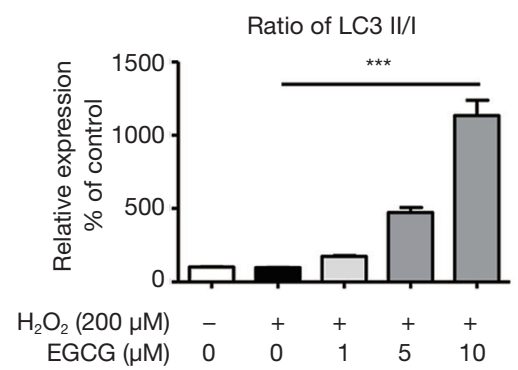

C

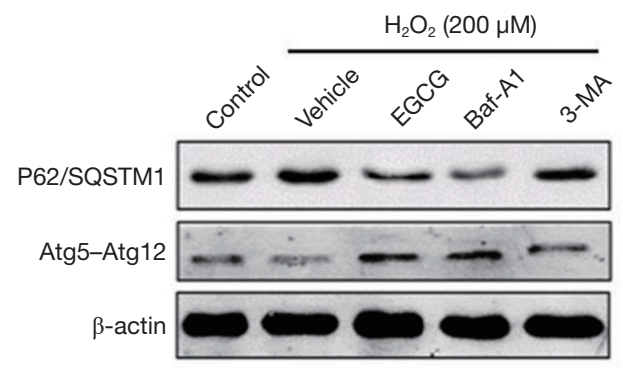

D
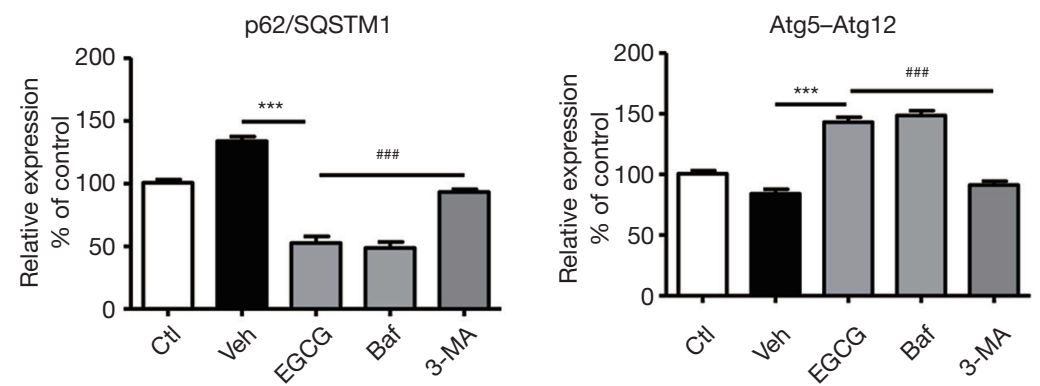

Figure 4 EGCG regulated expression of autophagy-related marker proteins. (A) Western blot analysis of endogenous LC3, Atg5 and Atg7 after challenge with EGCG at different concentrations $\left(1,5,10 \mu \mathrm{mol} / \mathrm{L}\right.$ ) for $24 \mathrm{~h}$ and $\mathrm{H}_{2} \mathrm{O}_{2}$ for $4 \mathrm{~h}$. (B) Bar charts show the quantification of Atg7, Atg5 and endogenous LC3-II/I. $\mathrm{n}=3 .{ }^{*} \mathrm{P}<0.05$, ${ }^{* *} \mathrm{P}<0.01$, ${ }^{* * *} \mathrm{P}<0.001$ vs. $\mathrm{H}_{2} \mathrm{O}_{2}$ alone group. (C) Western blot analysis of p62/SQSTM1 and Atg5-Atg12 complex after pretreated with EGCG (10 $\mathrm{mol} / \mathrm{L})$ and with or without 3-MA in HUVECs following $\mathrm{H}_{2} \mathrm{O}_{2}$ for $4 \mathrm{~h}$. (D) Bar charts show the quantification of p62/SQSTM1 and Atg5-Atg12 complex. Values are expressed as the mean $\pm \mathrm{SEM}, \mathrm{n}=3,{ }^{* * *} \mathrm{P}<0.001 v s$. $\mathrm{H}_{2} \mathrm{O}_{2}$ alone group. ${ }^{\prime \prime \prime \prime} \mathrm{P}<0.001$ vs. 3-MA pretreatment group. 
A $\mathrm{H}_{2} \mathrm{O}_{2}(200 \mu \mathrm{M})-+\quad+\quad+\quad+$ EGCG $(\mu \mathrm{M}) \quad 0 \quad 0 \quad 1 \quad 5 \quad 10$

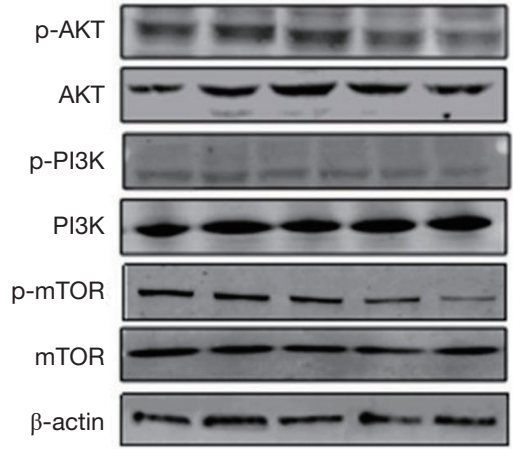

B

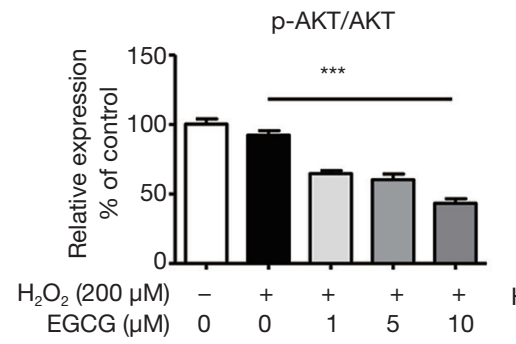

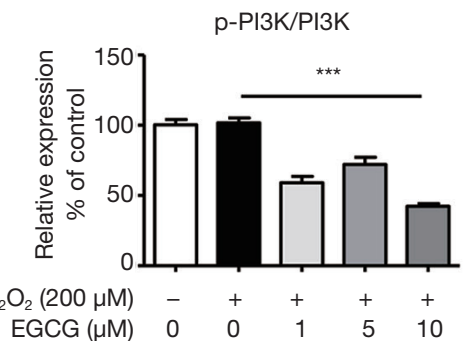

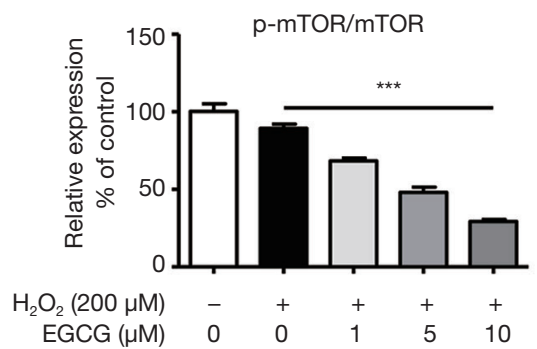

C

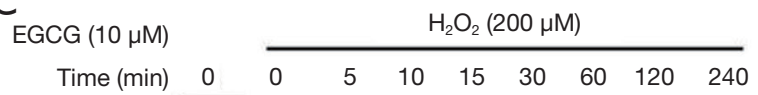

p-AKT

AKT

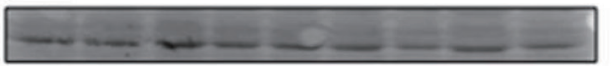

p-PI3K

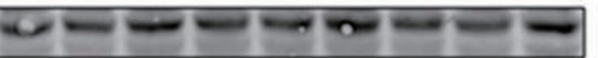

$\mathrm{PI} 3 \mathrm{~K}$

$\beta$-actin

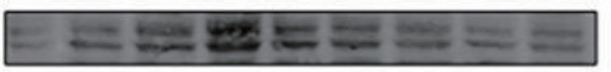

I3K

D
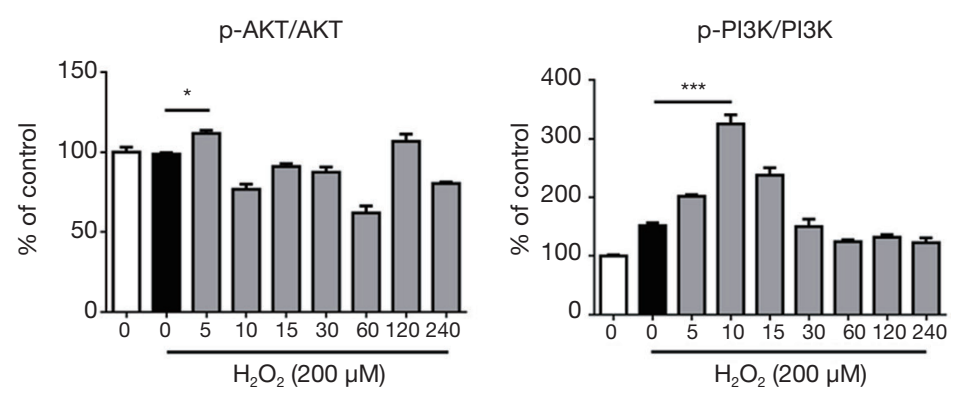

Figure 5 EGCG inhibited the PI3K-AKT-mTOR signaling pathway. (A) HUVECs with different concentrations $(1,5,10 \mu \mathrm{mol} / \mathrm{L})$ of EGCG for $24 \mathrm{~h}$ stimulated with $\mathrm{H}_{2} \mathrm{O}_{2}$ for $4 \mathrm{~h}$. (B) Bar charts show the quantification of p-AKT, p-PI3K, and p- mTOR. Values are expressed as the mean $\pm \mathrm{SEM}, \mathrm{n}=3$, ${ }^{* * *} \mathrm{P}<0.001$ vs. $\mathrm{H}_{2} \mathrm{O}_{2}$ alone group. (C) Lysates from HUVECs with EGCG $\left(10 \mu \mathrm{mol} / \mathrm{L}\right.$ ) for $24 \mathrm{~h}$ following $\mathrm{H}_{2} \mathrm{O}_{2}$ at different time points were analyzed by western blot for detection of the phosphorylation activity of AKT and PI3K. (D) The quantification of $\mathrm{p}$-AKT, p-PI3K at different time appoints. Values are expressed as the mean $\pm \mathrm{SEM}, \mathrm{n}=3,{ }^{*} \mathrm{P}<0.05,{ }^{* * *} \mathrm{P}<0.001$ vs. $\mathrm{H}_{2} \mathrm{O}_{2}$ alone group. 


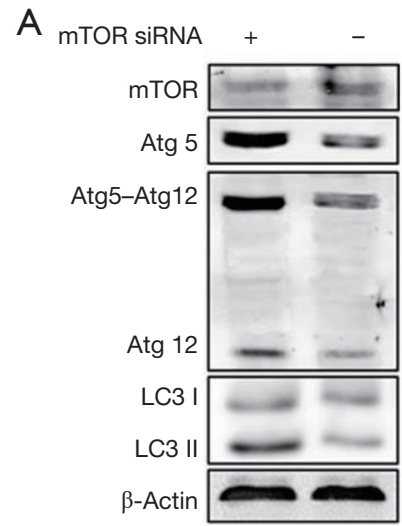

D

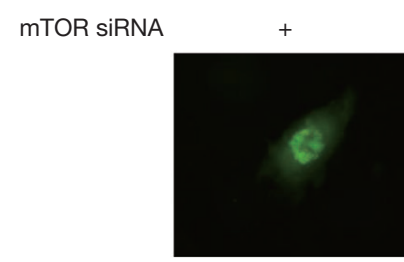

B

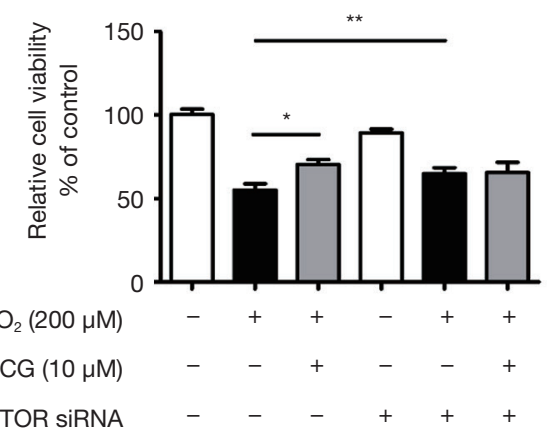

C

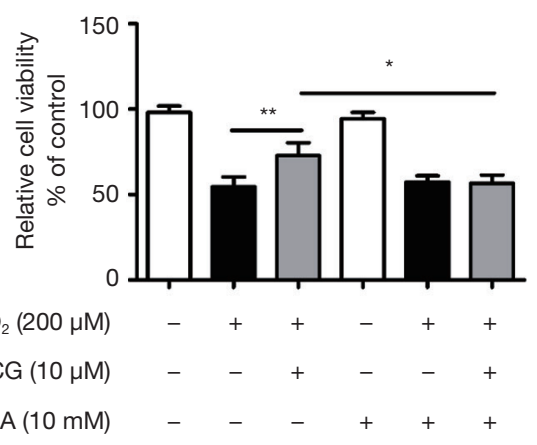

E
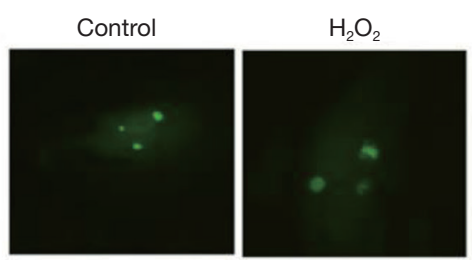

$\mathrm{H}_{2} \mathrm{O}_{2}+\mathrm{EGCG}$

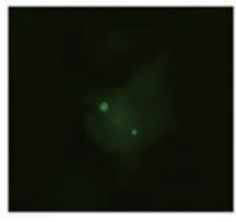

3-MA

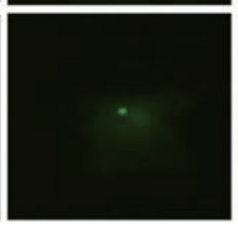

$\mathrm{H}_{2} \mathrm{O}_{2}+3-\mathrm{MA}$

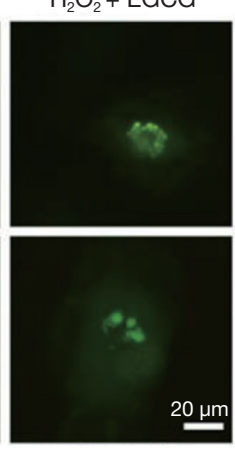

$\mathrm{H}_{2} \mathrm{O}_{2}+3-\mathrm{MA}+\mathrm{EGCG}$

Figure 6 Regulating autophagy interfered the protective effect of EGCG on HUVECs. (A) The knock down efficiency of mTOR siRNA on mTOR, Atg5, Atg12 and Atg5-Atg12 complex expression. (B) Representative image of LC3 puncta from $\mathrm{H}_{2} \mathrm{O}_{2}$ stimulation in HUVECs cells pretreated with EGCG $(10 \mu \mathrm{mol} / \mathrm{L})$ and with or without mTOR siRNA (48 h). $\mathrm{n}=3,{ }^{*}, \mathrm{P}<0.05,{ }^{* *}, \mathrm{P}<0.01$ vs. $\mathrm{H}_{2} \mathrm{O}_{2}$ alone group. $(\mathrm{C})$ EGCG $(10 \mu \mathrm{mol} / \mathrm{L})$ was pretreated on HUVECs for $24 \mathrm{~h}$ at different conditions of with or without mTOR siRNA (48 h). $\mathrm{n}=3$. **, $\mathrm{P}<0.01$ vs. $\mathrm{H}_{2} \mathrm{O}_{2}$ alone group. *, $\mathrm{P}<0.05$ vs. EGCG pretreated group. (D) Under the same conditions, 3-MA (10 mmol/L) was treated for 4 h. Values are presented as the means \pm SEM. (E) Representative image of LC3 puncta from $\mathrm{H}_{2} \mathrm{O}_{2}$ stimulation in HUVECs cells pretreated with EGCG (10 $\mu \mathrm{mol} / \mathrm{L})$ and with or without 3-MA (48 h).

Consistently, GFP-LC3 in HUVECs treated 3-MA showed a significant decrease (Figure 6E). Together, these data suggest that knockdown of mTOR partially promote EGCG-induced autophagy, whereas inhibiting autophagy in HUVECs can eliminate the cytoprotective effects of EGCG.

\section{Discussion}

It is well known that oxidative stress exhibits a critical role in many physiological and pathological processes in humans, including cancer, ischemic injury (19), chronic inflammation (6), type II diabetes (20) and arteriosclerosis (10,14,21-23). Thus, eliminating excess intracellular reactive oxygen species and preventing oxidative stress injury might be powerful interventions for the prevention and treatment of these diseases. EGCG, a polyphenol with antioxidant property belonging to the catechin family, is beneficial to human vascular endothelial, including myocyte hypertrophy, cardiac hypertrophy and fibrosis developing from aorta constriction (3). In this study, our data showed that EGCG could protect HUVECs from oxidative stress injury by inducing a beneficial autophagy.

Autophagy is an essential cellular mechanism which plays "housekeeping" role in normal physiological processes and defects in autophagy regulation play a central role in number of diseases. Autophagy can adapt cells to intracellular stress environmental, including oxidative stress, endoplasmic reticulum stress, starvation, hypoxia. Numerous studies have shown that autophagy acts primarily as a pro-survival process to protect cells from intracellular stress environmental, but sometimes, autophagy can also 
lead to cell death $(24,25)$. Besides, the relationship between apoptosis and autophagy has also been extensively studied. Apoptosis and autophagy can be initiated with similar stimuli, while in some case, they are initiated in a mutually exclusive manner (26). In our study, $\mathrm{H}_{2} \mathrm{O}_{2}$-induced apoptosis in HUVECs was reduced and autophagy was increased when pretreated with EGCG.

In recent studies, EGCG in high doses $(>40 \mu \mathrm{mol} / \mathrm{L})$ suppress cancer cells by inducing apoptosis and by inhibiting autophagic processes $(27,28)$. However, several studies also illustrate that EGCG in low doses $(<20 \mu \mathrm{mol} / \mathrm{L})$ play a preventive role in cancer developing via promoting autophagic processes during the early phase of cancer (29). Based on these studies, we observed that EGCG in low doses $(<20 \mu \mathrm{mol} / \mathrm{L})$ showed an autophagy-inductive effect on both cancer cells and normal cells. In our study results, we found that EGCG inhibited the apoptosis process at a concentration of $10 \mu \mathrm{mol} / \mathrm{L}$, but the effect was not strong enough to completely protect HUVECs from oxidative stress-caused cell death. Since EGCG is a multi-target compound, we can confirm that the protective effect of EGCG on $\mathrm{H}_{2} \mathrm{O}_{2}$-caused HUVECs cell death is not only based a single mechanism.

In the following studies, we analyzed the underlying molecular mechanisms involved in EGCG-mediated autophagy induction. We know that the PI3K-AKT-mTOR signaling pathway plays an essential role in many biological and pathological processes in cancer cells and normal cells (30-32). mTOR is one essential regulator of autophagy because of its ability to sense stress, growth factor and nutrient states (33). We all know that mTOR signaling is very vital in regulating lysosomal function (34). It has been confirmed that EGCG competitively inhibits PI3K and mTOR with Ki values of 380 and $320 \mathrm{nM}$, respectively (35). We found that EGCG down-regulated phosphorylation of mTOR in a dose dependent manner, which is consistent with the opinion that mTOR mediates autophagy by regulating phosphorylation (36). Knockdown of mTOR partially promoted the protective effect of EGCG on oxidative stress. This result provided solid evidence on our hypothesis that EGCG reverses $\mathrm{H}_{2} \mathrm{O}_{2}$-caused damage on HUVECs via targeting mTOR.

\section{Conclusions}

In this work, we illustrated EGCG in low doses can protect vascular endothelial cells from oxidative stress-induced damage by inducing the autophagy and inhibiting apoptosis.
In particular, pretreatment with EGCG significantly improved the survival of HUVECs from $\mathrm{H}_{2} \mathrm{O}_{2}$-induced cell death, upregulated the levels of Atg 5, Atg 7, LC3 II/I, and the Atg5-Atg12 complex and downregulated apoptosisrelated proteins expression. In addition, we verified that EGCG induced autophagy by targeting the PI3K-AKTmTOR pathway. All of these results indicate a novel role for EGCG in the treatment of oxidative stress-related cardiovascular and cerebrovascular diseases.

\section{Acknowledgments}

Funding: This work was supported by the Shaanxi Fourth People's Hospital (grant number: 2017SY-003). We are grateful to Prof. Li for kindly donated the mCherry-EGFPLC3 plasmid.

\section{Footnote}

Conflicts of Interest: The authors have no conflicts of interest to declare.

Ethical Statement: The authors are accountable for all aspects of the work in ensuring that questions related to the accuracy or integrity of any part of the work are appropriately investigated and resolved. All animal experiments were performed according to NIH Guide for the Care and Use of Laboratory Animals and approved by the Institutional Animal Care and Use Committee of the Fourth People's Hospital of Shaanxi (China).

Open Access Statement: This is an Open Access article distributed in accordance with the Creative Commons Attribution-NonCommercial-NoDerivs 4.0 International License (CC BY-NC-ND 4.0), which permits the noncommercial replication and distribution of the article with the strict proviso that no changes or edits are made and the original work is properly cited (including links to both the formal publication through the relevant DOI and the license). See: https://creativecommons.org/licenses/by-nc-nd/4.0/.

\section{References}

1. Maiese K, Chong ZZ, Hou J, et al. Oxidative stress: Biomarkers and novel therapeutic pathways. Exp Gerontol 2010;45:217-34.

2. Johnson R, Bryant S, Huntley AL. Green tea and green tea catechin extracts: an overview of the clinical evidence. 
Maturitas 2012;73:280-7.

3. Khurana S, Venkataraman K, Hollingsworth A, et al. Polyphenols: benefits to the cardiovascular system in health and in aging. Nutrients 2013;5:3779-827.

4. Meydani M, Hasan ST. Dietary polyphenols and obesity. Nutrients 2010;2:737-51.

5. Shankar S, Ganapathy S, Srivastava RK. Green tea polyphenols: biology and therapeutic implications in cancer. Front Biosci 2007;12:4881-99.

6. Fürst R, Zundorf I. Plant-derived anti-inflammatory compounds: hopes and disappointments regarding the translation of preclinical knowledge into clinical progress. Mediators Inflamm 2014;2014:146832.

7. Ge D, Jing Q, Meng N, et al. Regulation of apoptosis and autophagy by sphingosylphosphorylcholine in vascular endothelial cells. J Cell Physiol 2011;226:2827-33.

8. Mizushima N, Levine B, Cuervo AM, et al. Autophagy fights disease through cellular self-digestion. Nature 2008;451:1069-75.

9. Kimura S, Noda T, Yoshimori T. Dissection of the autophagosome maturation process by a novel reporter protein, tandem fluorescent-tagged LC3. Autophagy 2007;3:452-60.

10. Jain SK, McVie R, Duett J, et al. Erythrocyte membrane lipid peroxidation and glycosylated hemoglobin in diabetes. Diabetes 1989;38:1539-43.

11. Xie Y, You SJ, Zhang YL, et al. Protective role of autophagy in AGE-induced early injury of human vascular endothelial cells. Mol Med Rep 2011;4:459-64.

12. Lemaître V, Dabo AJ, D'Armiento J. Cigarette smoke components induce matrix metalloproteinase- 1 in aortic endothelial cells through inhibition of mTOR signaling. Toxicol Sci 2011;123:542-9.

13. Mancini D, Pinney S, Burkhoff D, et al. Use of rapamycin slows progression of cardiac transplantation vasculopathy. Circulation 2003;108:48-53.

14. Mueller CF, Laude K, McNally JS, et al. ATVB in focus: redox mechanisms in blood vessels. Arterioscler Thromb Vasc Biol 2005;25:274-8.

15. Jaffe EA, Nachman RL, Becker CG, et al. Culture of human endothelial cells derived from umbilical veins. Identification by morphologic and immunologic criteria. J Clin Invest 1973;52:2745-56.

16. Pan Y, Zhong LJ, Zhou H, et al. Roles of vimentin and 143-3 zeta/delta in the inhibitory effects of heparin on PC$3 \mathrm{M}$ cell proliferation and B16-F10-luc-G5 cells metastasis. Acta Pharmacol Sin 2012;33:798-808.

17. Klionsky DJ, Abeliovich H, Agostinis P, et al. Guidelines for the use and interpretation of assays for monitoring autophagy in higher eukaryotes. Autophagy 2008;4:151-75.

18. Pankiv S, Clausen TH, Lamark T, et al. p62/SQSTM1 binds directly to Atg8/LC3 to facilitate degradation of ubiquitinated protein aggregates by autophagy. J Biol Chem 2007;282:24131-45.

19. Zhu S, Li W, Ward MF, et al. High mobility group box 1 protein as a potential drug target for infection- and injuryelicited inflammation. Inflamm Allergy Drug Targets 2010;9:60-72

20. Munir KM, Chandrasekaran S, Gao F, et al. Mechanisms for food polyphenols to ameliorate insulin resistance and endothelial dysfunction: therapeutic implications for diabetes and its cardiovascular complications. Am J Physiol Endocrinol Metab 2013;305:E679-86.

21. Brownlee M. Biochemistry and molecular cell biology of diabetic complications. Nature 2001;414:813-20.

22. Fouraux MA, Deneka M, Ivan V, et al. Rabip4' is an effector of rab5 and rab4 and regulates transport through early endosomes. Mol Biol Cell 2004;15:611-24.

23. Vaziri ND, Rodriguez-Iturbe B. Mechanisms of disease: oxidative stress and inflammation in the pathogenesis of hypertension. Nat Clin Pract Nephrol 2006;2:582-93.

24. Levine B, Yuan J. Autophagy in cell death: an innocent convict? J Clin Invest 2005;115:2679-88.

25. Galluzzi L, Bravo-San Pedro JM, Levine B, et al. Pharmacological modulation of autophagy: therapeutic potential and persisting obstacles. Nat Rev Drug Discov 2017;16:487-511.

26. Lum JJ, DeBerardinis RJ, Thompson CB. Autophagy in metazoans: cell survival in the land of plenty. Nat Rev Mol Cell Biol 2005;6:439-48.

27. Chen L, Ye HL, Zhang G, et al. Autophagy inhibition contributes to the synergistic interaction between EGCG and doxorubicin to kill the hepatoma Hep3B cells. PLoS One 2014;9:e85771.

28. Lecumberri E, Dupertuis YM, Miralbell R, et al. Green tea polyphenol epigallocatechin-3-gallate (EGCG) as adjuvant in cancer therapy. Clin Nutr 2013;32:894-903.

29. Irimie AI, Braicu C, Zanoaga O, et al. Epigallocatechin-3gallate suppresses cell proliferation and promotes apoptosis and autophagy in oral cancer SSC-4 cells. Onco Targets Ther 2015;8:461-70.

30. Cui H, Wu S, Shang Y, et al. Pleurotus nebrodensis polysaccharide(PN50G) evokes A549 cell apoptosis by the ROS/AMPK/PI3K/AKT/mTOR pathway to suppress tumor growth. Food Funct 2016;7:1616-27.

31. Xiaokaiti Y, Wu H, Chen Y, et al. EGCG reverses human 
Page 12 of 12

neutrophil elastase-induced migration in A549 cells by directly binding to HNE and by regulating alpha1-AT. Sci Rep 2015;5:11494.

32. Yang Y, Cong H, Han C, et al. 12-Deoxyphorbol 13-palmitate inhibits the expression of VEGF and HIF1alpha in MCF-7 cells by blocking the PI3K/Akt/mTOR signaling pathway. Oncol Rep 2015;34:1755-60.

33. Jung CH, Ro SH, Cao J, et al. mTOR regulation of autophagy. FEBS Lett 2010;584:1287-95.

34. Sinha D, Valapala M, Shang P, et al. Lysosomes: Regulators
Meng et al. EGCG protects HUVECs from ROS-induced damage

of autophagy in the retinal pigmented epithelium. Exp Eye Res 2016;144:46-53.

35. Van Aller GS, Carson JD, Tang W, et al. Epigallocatechin gallate (EGCG), a major component of green tea, is a dual phosphoinositide-3-kinase/mTOR inhibitor. Biochem Biophys Res Commun 2011;406:194-9.

36. Alers S, Loffler AS, Wesselborg S, et al. Role of AMPKmTOR-Ulk1/2 in the regulation of autophagy: cross talk, shortcuts, and feedbacks. Mol Cell Biol 2012;32:2-11.

Cite this article as: Meng J, Chen Y, Wang J, Qiu J, Chang C, Bi F, Wu X, Liu W. EGCG protects vascular endothelial cells from oxidative stress-induced damage by targeting the autophagy-dependent PI3K-AKT-mTOR pathway. Ann Transl Med 2020;8(5):200. doi: 10.21037/atm.2020.01.92 